\title{
Reusable polymer-supported catalyst for the [3+2] Huisgen cycloaddition in automation protocols
}

\author{
Christian Girard, ${ }^{\star}$ Esra Önen, Marie Aufort, Sophie Beauvière, Edmond Samson and Jean Herscovici \\ Unité de Pharmacologie Chimique \& Génétique: Synthèse, Imagerie et Electrochimie, Ecole Nationale Supérieure de \\ Chimie de Paris, 11 rue Pierre et Marie Curie, 75005 Paris, France. Inserm, U640, Paris, F-75006 France; CNRS, \\ UMR8151, Paris, F-75006 France
}

christian-girard@enscp.fr

SUPPORTING INFORMATION

\section{General Methods}

Chemicals: Copper (I) idodide, propiolic acid methyl ester and propiolaldehyd diethyl acetal were purchased from Lancaster, propargyl alcohol from Aldrich and used without further purification. Propargyl phthalimide and phenyl ether were prepared from propargyl bromide and potassium phthalimide [Glacet, C.; Coupé, R. Bull. Soc. Chim. Fr. 1963, 2464. Müller, P.; Imogaï, H. Tetrahedron : Asym. 1998, 9, 4419.] and phenol respectively [Pourcelot, G.; Cadiot, P. Bull. Soc. Chim. Fr. 1966, 3016.]. Azides were prepared from sodium azide and benzyl bromide, 3-chloropropanol and ethyl bromoacetate following published procedures [Alvarez, S. G.; Alvarez, M. T. Synthesis 1997, 413. Hooper, N.; Beeching, L. J.; Dyke, J. M.; Morris, A.; Ogden, J. S.; Dias, A. A.; Costa, M. L.; Barros, M. T.; Cabrell, M. H.; Moutinho, A. M. C. J. Phys. Chem. A. 2002, 106, 9968. Scheel, A. J., Komber, H.; Voit, B. I. Macromol. Rapid Commun 2004, 25, 1175].

Solvents: Acetonitrile (spectrometric grade, low water) was purchased from SDS and used as such. Dichloromethane was treated with phosphorus pentaoxide at reflux $(1 \mathrm{~h})$ before being distilled.

Chromatographic purifications: Chromatographic separations were performed on SDS $6035-70 \mu \mathrm{m}$ silica gel on short pads (compound/silica $=1 / 5)$ in polyethylene $(\mathrm{PE})$ tubes or serynges equipped with a PE frit using pressure. The pad was washed with heptane, the compound loaded in a minimum of dichloromethane, and the chromatography performed using a gradient from pure heptane to ethyl acetate.

Nuclear Magnetic Resonance (NMR): NMR spectra were recorded on a Bruker Avance DRX in deuteriochloroform at $300 \mathrm{MHz}$ for the ${ }^{1} \mathrm{H}$ and 75,5 for the ${ }^{13} \mathrm{C}$. Chemical shifts $(\delta)$ are reported in part per million ( $\mathrm{ppm}$ ) relative to to the tetramethylsilane signal as an internal reference. Couplings constants $(J)$ are in hertz and signal multiplicities indicated as s (singulet), d (doublet), t (triplet), q (quadruplet), m (multiplet), dd (doublet of doublet).

Liquid Chromatography / Mass Spectrometry (LC-MS): LC-MS analyses were done on a Shimadzu LCSM-2010 A on a HPLC, column Alltima HP C8 $3 \mu$ (Alltech), reversed phase (L= $53 \mathrm{~mm}$; ID $=7 \mathrm{~mm})$, PDA diodes detector SPD-M10 A ( $\mathrm{D}_{2}$, lamp from 190 to $400 \mathrm{~nm}$ ) and light scattering dectector ELSD-LT. The LC were runned using a $1 \mathrm{ml} / \mathrm{min}$ flow using a gradient between acetonitrile and water containing formic acid (0,1\%): 0 to $1 \mathrm{~min}: 30 \% \mathrm{CH}_{3} \mathrm{CN}, 1$ to $5 \mathrm{~min}$ : from $30 \%$ to $100 \%$ $\mathrm{CH}_{3} \mathrm{CN}, 5$ to $12 \mathrm{~min}: 100 \% \mathrm{CH}_{3} \mathrm{CN}, 12$ to $14,99 \mathrm{~min}$ : from $100 \%$ to $30 \% \mathrm{CH}_{3} \mathrm{CN}, 14,99$ to $20 \mathrm{~min}$ : $30 \% \mathrm{CH}_{3} \mathrm{CN}$. MS was recorded between $\mathrm{m} / \mathrm{z}=100$ to 500 at the exit of the colum using a ESI ionization and positive ion mode (detector $=1.5 \mathrm{kV}$, quadripole $=5 \mathrm{~V}$ ). 


\section{$\underline{\text { Procedures }}$}

Dry Amberlyst A-21: Commercial wet Amberlyst A-21 resin (20-50 mesh, $100 \mathrm{~g}$ ) was suspended in $\mathrm{MeOH}(500 \mathrm{~mL})$ for $0.5 \mathrm{~h}$ and filtered (3 times) and then soaked in methylene chloride $(500 \mathrm{~mL})$ for $0.5 \mathrm{~h}$ and again filtered (3 times). The resulting resin was placed in a round-bottom flask on a rotoevaporator and dried at $50{ }^{\circ} \mathrm{C}$ under $10 \mathrm{~mm} \mathrm{Hg}$ until it was free-flowing. The dried resin was then kept overnight in vacuo in a desiccator over $\mathrm{P}_{2} \mathrm{O}_{5}$. Specifications from the manufacturer indicate that the polymer contains 4.8 mequiv of amine/g of dry resin.

Preparation of the supported catalyst (A-21.CuI): Dry Amberlyst A-21 (1.0 g, 4.8 mmol amine) was added to a solution of copper (I) iodide $(381 \mathrm{mg}, 2.00 \mathrm{mmol})$ in acetonitrile $(15 \mathrm{ml})$ and gently shaken on an orbital stirrer for $17 \mathrm{~h}$. The solvent was drawn off and the resin washed with $\mathrm{CH}_{3} \mathrm{CN}(2 \times 15 \mathrm{ml})$, $\mathrm{CH}_{2} \mathrm{Cl}_{2}(2 \times 15 \mathrm{ml})$ and dried in vacuo $(0.01 \mathrm{~mm} \mathrm{Hg})$ at $40^{\circ} \mathrm{C}$. The weight increase was of $0,307 \mathrm{~g}(1.61$ mmol CuI) which gave the polymer a loading of $1.23 \mathrm{mmol} \mathrm{CuI.g^{-1 }}$.

General procedure for the triazole synthesis: The polymer supported catalyst (Amberlyst A-21•CuI, $1.23 \mathrm{mmol} / \mathrm{g}, 31 \mathrm{mg}, 0.038 \mathrm{mmol}, 13 \% \mathrm{~mol}$ ) was placed in a series of reactors on a Chemspeed ASW2000. The azides $(0.55 \mathrm{mmol})$ dissolved in $1 \mathrm{~mL}$ of $\mathrm{CH}_{2} \mathrm{Cl}_{2}$ were added dropwise followed by a solution of the alkynes $(0.50 \mathrm{mmol})$ in $1 \mathrm{~mL}$ of $\mathrm{CH}_{2} \mathrm{Cl}_{2}$. The suspension was orbitally stirred $(600 \mathrm{rpm})$ for $12 \mathrm{~h}$ at room temperature. The solutions were filtered and the reactors and polymers were washed with $\mathrm{CH}_{2} \mathrm{Cl}_{2}(2 \times 2 \mathrm{~mL})$. The extracts were evaporated under reduced pressure and the products dried in vacuo.

\section{$\underline{\text { Results and Analyses }}$}

2-(1-Benzyl-1H-[1,2,3]triazol-4-ylmethyl)isoindole-1,3-dione (9):

$\mathrm{C}_{18} \mathrm{H}_{14} \mathrm{~N}_{4} \mathrm{O}_{2}, \mathrm{M}=318.34$ g.mol ${ }^{-1}$

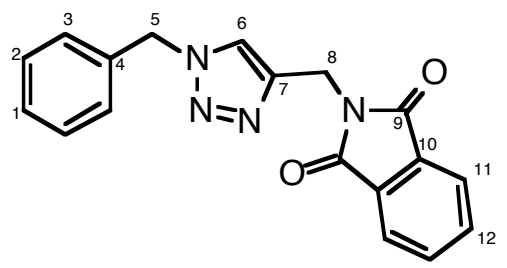

Prepared from $93 \mathrm{mg}(0.50 \mathrm{mmol})$ propargyl phthalimide (1) and $73 \mathrm{mg}(0.55 \mathrm{mmol})$ benzyl azide (6). The product 9 was obtained as a white solid (148 mg, $93 \%)$.

$\mathrm{mp}=179-181^{\circ} \mathrm{C}$

${ }^{1} \mathbf{H}$ NMR $\left(\mathbf{C D C l}_{3}\right) \delta 4.97$ (s, 2H, H-8), 5.49 (s, 2H, H-5), 7.25-7.37 (m, 5H, H-1, H-2, H-3), 7.51 (s, 1H, H-6), 7.70-7.85 (m, 4H, H-11, H-12)

${ }^{13} \mathbf{C ~ N M R}\left(\mathbf{C D C l}_{3}\right) \delta 33.1(\mathrm{C}-8), 54.2(\mathrm{C}-5), 122.7$ (C-1), 123.4 (C-11), 128.1 (C-2), 128.7 (C-3), 129.1 5c-4), 132.0 (C-12), 134.1 (C-10), 134.5 (C-6), 143.1 (C-7), 167.6 (C-9)

LC-MS : ELSD 98\%, $\mathrm{R}_{\mathrm{t}}=9.10 \mathrm{~min} ., \mathrm{m} / \mathrm{z} 319(\mathrm{M}+\mathrm{H})^{+}$ 


\section{1-Benzyl-4-(phenoxymethyl)triazole (10):}

$\mathrm{C}_{16} \mathrm{H}_{15} \mathrm{~N}_{3} \mathrm{O}, \mathrm{M}=265.31 \mathrm{~g} . \mathrm{mol}^{-1}$<smiles></smiles>

Prepared from $66 \mathrm{mg}(0.50 \mathrm{mmol})$ propargyl phenyl ether (2) and $73 \mathrm{mg}(0.55 \mathrm{mmol})$ benzyl azide (6). The product 10 was obtained as a white solid (122 mg, $92 \%)$.

$\mathrm{mp}=119-121^{\circ} \mathrm{C}$

${ }^{1} \mathbf{H}$ NMR $\left(\mathbf{C D C l}_{3}\right) \delta 5.18$ (s, 2H, H-8), 5.51 (s, 2H, H-5), 6.90-7.39 (m, 10H, Harom), 7.52 (s, 1H, H-6) ${ }^{13} \mathbf{C ~ N M R}\left(\mathbf{C D C l}_{3}\right) \delta 54.2(\mathrm{C}-5), 62.0(\mathrm{C}-8), 114.8(\mathrm{C}-10), 121.3$ (C-1), 128.1 (C-2), $128.8(\mathrm{C}-3), 129.1$ (C-4), 129.5 (C-11), 134.5 (C-6), 144.6 (C-7), 158.2 (C-9)

LC-MS : ELSD 92\%, $\mathrm{R}_{\mathrm{t}}=9.56 \mathrm{~min} ., \mathrm{m} / \mathrm{z} 266(\mathrm{M}+\mathrm{H})^{+}$

(1-Benzyltriazol-4-yl)methanol (11):

$\mathrm{C}_{10} \mathrm{H}_{11} \mathrm{~N}_{3} \mathrm{O}, \mathrm{M}=189.22 \mathrm{~g} \cdot \mathrm{mol}^{-1}$

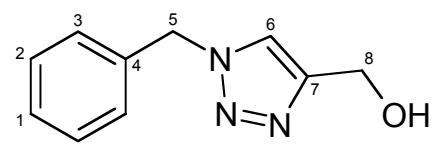

Prepared from $28 \mathrm{mg}(0.50 \mathrm{mmol})$ propargyl alcohol (3) and $73 \mathrm{mg}(0.55 \mathrm{mmol})$ benzyl azide (6). The product 11 was obtained as a white solid (92 $\mathrm{mg}, 97 \%)$.

$\mathrm{mp}=76-78^{\circ} \mathrm{C}\left(\right.$ litt. $\left.^{1} 76-77{ }^{\circ} \mathrm{C}\right)$

${ }^{1} \mathbf{H}$ NMR $\left(\mathbf{C D C l}_{3}\right) \delta 4.47$ (s, 1H, OH), 4.70(s, 2H, H-8), 5.46 (s, 2H, H-5), 7.17-7.45 (m, 5 H, Harom), $7.91(\mathrm{~s}, 1 \mathrm{H}, \mathrm{H}-6)$

${ }^{13} \mathbf{C}$ NMR $\left(\mathbf{C D C l}_{3}\right) \delta 54.1(\mathrm{C}-5), 56.0(\mathrm{C}-8), 122.0(\mathrm{C}-1), 128.1(\mathrm{C}-2), 128.7(\mathrm{C}-3), 129.1(\mathrm{C}-4), 134.5$ (C-6), $148.0(\mathrm{C}-7)$

LC-MS : ELSD 97\%, $\mathrm{R}_{\mathrm{t}}=3.41 \mathrm{~min} ., \mathrm{m} / \mathrm{z}$ 190(M+H $)^{+}$

\section{1-Benzyl-4-(diethoxymethyl)triazole (12):}

$\mathrm{C}_{14} \mathrm{H}_{19} \mathrm{~N}_{3} \mathrm{O}_{2}, \mathrm{M}=261.33$ g. $\mathrm{mol}^{-1}$

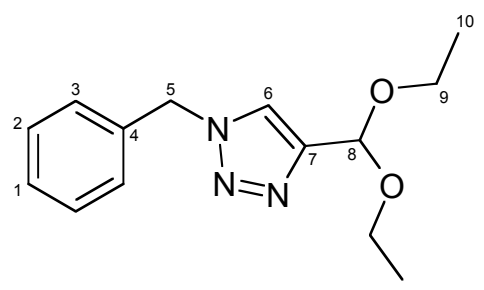

Prepared from $64 \mathrm{mg}(0.50 \mathrm{mmol})$ propiolaldehyde dimethyl acetal (4) and $73 \mathrm{mg}(0.55 \mathrm{mmol})$ benzyl azide (6). The product 12 was obtained as a white solid (129 mg, $99 \%)$.

$\mathrm{mp}=58-60{ }^{\circ} \mathrm{C}$

1. De la Heras, F. G.; Alonso, R.; Alonso, G. J. Med. Chem. 1979, 22, 496. 
${ }^{1} \mathbf{H}$ NMR $\left(\mathbf{C D C l}_{3}\right) \delta 1.20(\mathrm{t}, J=7.0 \mathrm{~Hz}, 6 \mathrm{H}, \mathrm{H}-10) 3.62(2 \mathrm{q}, J=7.1 \mathrm{~Hz}, 4 \mathrm{H}, \mathrm{H}-9), 5.50(\mathrm{~s}, 2 \mathrm{H}, \mathrm{H}-5), 5.68$ (s, 1H, H-8), 7.25-7.35 (m, 5H, H-arom), 7.49(s, 1H, H-6)

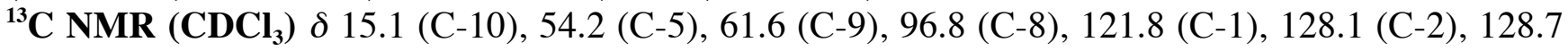
(C-3), 129.1 (C-4), 134.5 (C-6), 147.5 (C-7)

LC-MS : ELSD 89\%, $\mathrm{R}_{\mathrm{t}}=8.88 \mathrm{~min} ., \mathrm{m} / \mathrm{z} 216(\mathrm{M}-\mathrm{EtO})$

\section{Methyl 1-benzyltriazole-4-carboxylate (13):}

$\mathrm{C}_{11} \mathrm{H}_{11} \mathrm{~N}_{3} \mathrm{O}_{2}, \mathrm{M}=217.23 \mathrm{~g} \cdot \mathrm{mol}^{-1}$<smiles>COC(=O)c1cn(Cc2ccccc2)nn1</smiles>

Prepared from $42 \mathrm{mg}(0.50 \mathrm{mmol})$ propiolic acid methyl ester (5) and $73 \mathrm{mg}(0.55 \mathrm{mmol})$ benzyl azide (6). The product 13 was obtained as a white off-solid (107 mg, $99 \%$ ).

$\mathrm{mp}=116-118^{\circ} \mathrm{C}\left(\right.$ litt. $\left.^{2} 115-116^{\circ} \mathrm{C}\right)$

${ }^{1} \mathbf{H}$ NMR $\left(\mathbf{C D C l}_{3}\right) \delta 3.90$ (s, 3H, H-9), 5.55 (s, 2H, H-5), 7.27-7.36 (m, 5H, H-arom), 7.99 (s, 1H, H-6)

${ }^{13} \mathbf{C ~ N M R}\left(\mathbf{C D C l}_{3}\right) \delta 52.2$ (C-9), 54.5 (C-5), 127.3 (C-1), 128.3 (C-2), 129.2 (C-3), 129.3 (C-4), 133.6 (C-7), $140.3(\mathrm{C}-6), 161.1(\mathrm{C}-8)$

LC-MS : ELSD $89 \%, \mathrm{R}_{\mathrm{t}}=8.88 \mathrm{~min} ., \mathrm{m} / \mathrm{z} 218(\mathrm{M}+\mathrm{H})^{+}$

2-[[1-(3-Hydroxypropyl)triazol-4-yl]methyl]isoindoline-1,3-dione (14):

$\mathrm{C}_{14} \mathrm{H}_{14} \mathrm{~N}_{4} \mathrm{O}_{3}, \mathrm{M}=286.11$ g.mol ${ }^{-1}$<smiles>O=C1c2ccccc2C(=O)N1Cc1cn(CCCO)nn1</smiles>

Prepared from $93 \mathrm{mg}(0.50 \mathrm{mmol})$ propargyl phthalimide (1) and $56 \mathrm{mg}(0.55 \mathrm{mmol})$ propanol azide (7). The product 14 was obtained as a white solid (87 $\mathrm{mg}, 61 \%)$.

$\mathrm{mp}=116-119^{\circ} \mathrm{C}$

${ }^{1} \mathbf{H}$ NMR $\left(\mathbf{C D C l}_{3}\right) \delta 2.07(\mathrm{q}, J=6.0 \mathrm{~Hz}, 2 \mathrm{H}, \mathrm{H}-2), 3.61(\mathrm{t}, J=6.0 \mathrm{~Hz}, 2 \mathrm{H}, \mathrm{H}-1), 4.47(\mathrm{t}, J=6.0 \mathrm{~Hz}, 2 \mathrm{H}$, $\mathrm{H}-3), 4.96$ (s, 2H, H-6), 7.68-7.71 (m, 2H, H-arom), 7.81-7.83 (m, 2H, H-arom).

${ }^{13} \mathbf{C}$ NMR $\left(\mathbf{C D C l}_{3}\right) \delta 32.5(\mathrm{C}-2), 33.0(\mathrm{C}-6), 46.9$ (C-3), 58.8 (C-1), $123.2(\mathrm{C}-4), 123.4(\mathrm{C}-9), 132.0$ (C10), 134.1 (C-8), $167.7(\mathrm{C}-7)$

LC-MS : ELSD 99\%, $\mathrm{R}_{\mathrm{t}}=3.31 \mathrm{~min} ., \mathrm{m} / \mathrm{z} 287(\mathrm{M}+\mathrm{H})^{+}$

2.Abu-Orabi, S. T.; Atfah, A.; Jibril, I.; Al-Sheik Ali, A. Marii, F. Gazz. Chim. Ital. 1992, 122, 29. 
3-(4-Phenoxytriazol-1-yl)propan-1-ol (15):

$\mathrm{C}_{12} \mathrm{H}_{15} \mathrm{~N}_{3} \mathrm{O}_{2}, \mathrm{M}=233.27$ g.mol ${ }^{-1}$

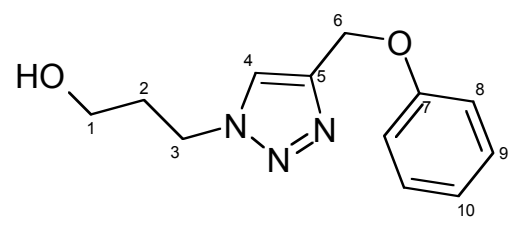

Prepared from $66 \mathrm{mg}(0.50 \mathrm{mmol})$ propargyl phenyl ether $(2)$ and $56 \mathrm{mg}(0.55 \mathrm{mmol})$ propanol azide (7). The product 15 was obtained as a off-white solid (105 $\mathrm{mg}, 90 \%)$.

$\mathrm{mp}=39-41{ }^{\circ} \mathrm{C}$

${ }^{1} \mathbf{H}$ NMR $\left(\mathbf{C D C l}_{3}\right) \delta 2.10(\mathrm{q}, J=6.0 \mathrm{~Hz}, 2 \mathrm{H}, \mathrm{H}-2), 3.23(\mathrm{~s}, 1 \mathrm{H}, \mathrm{OH}), 3.62(\mathrm{t}, J=6.0 \mathrm{~Hz}, 2 \mathrm{H}, \mathrm{H}-1), 4.49$ (t, J=6.0 Hz, 2H, H-3), 5.16 (s, 2H, H-6), 6.94-6.97 (m, 3H, H-arom), 7.24-7.30 (m, 2H, H-arom), 7.66 (s, $1 \mathrm{H}, \mathrm{H}-4)$

${ }^{13} \mathbf{C}$ NMR $\left(\mathbf{C D C l}_{3}\right) \delta 32.6(\mathrm{C}-2), 47.1(\mathrm{C}-3), 58.5$ (C-1), 61.9 (C-6), 114.7 (C-8), 121.3 (C-10), 123.2 (C-4), 129.5 (C-9), 144.1 (C-5), $158.2(\mathrm{C}-7)$

LC-MS : ELSD 99\%, $\mathrm{R}_{\mathrm{t}}=4.08 \mathrm{~min} ., \mathrm{m} / \mathrm{z} 234(\mathrm{M}+\mathrm{H})^{+}, 256(\mathrm{M}+\mathrm{Na})^{+}$

\section{3-[4-(Hydroxymethyl)triazol-1-yl]propan-1-ol (16):}

$\mathrm{C}_{6} \mathrm{H}_{11} \mathrm{~N}_{3} \mathrm{O}_{2}, \mathrm{M}=157.17$ g. $\mathrm{mol}^{-1}$

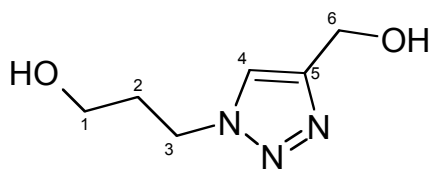

Prepared from $28 \mathrm{mg}(0.50 \mathrm{mmol})$ propargyl alcohol (3) and $56 \mathrm{mg}(0.55 \mathrm{mmol})$ propanol azide (7). The product 16 was obtained as a viscous colorless oil (10 $\mathrm{mg}, 13 \%)$.

${ }^{1} \mathbf{H}$ NMR $\left(\left(\mathbf{C D}_{3}\right)_{2} \mathbf{C}(\mathbf{0})\right) \delta 2.02(\mathrm{q}, J=6.0 \mathrm{~Hz}, 2 \mathrm{H}, \mathrm{H}-2) 3.24(\mathrm{~s}, 1 \mathrm{H}, \mathrm{OH}), 3.49(\mathrm{t}, J=6.0 \mathrm{~Hz}, 2 \mathrm{H}, \mathrm{H}-1)$, $4.41(\mathrm{t}, J=6.0 \mathrm{~Hz}, 2 \mathrm{H}, \mathrm{H}-3), 4.63$ (s, 2H, H-6), 7.66( s, $1 \mathrm{H}, \mathrm{H}-4)$

${ }^{13} \mathbf{C}$ NMR $\left(\left(\mathbf{C D}_{3}\right)_{2} \mathbf{C}(\mathbf{0})\right) \delta 33.8(\mathrm{C}-2), 47.5(\mathrm{C}-3), 56.5(\mathrm{C}-6), 58.8(\mathrm{C}-1), 122.8(\mathrm{C}-4), 148.9(\mathrm{C}-5)$

LC-MS : ELSD 90\%, $\mathrm{R}_{\mathrm{t}}=2.67 \mathrm{~min} ., \mathrm{m} / \mathrm{z} 158(\mathrm{M}+\mathrm{H})^{+}$

\section{3-[4-(Diethoxymethyl)triazol-1-yl]propan-1-ol (17):}

$\mathrm{C}_{10} \mathrm{H}_{19} \mathrm{~N}_{3} \mathrm{O}_{3}, \mathrm{M}=229.28 \mathrm{~g} \cdot \mathrm{mol}^{-1}$

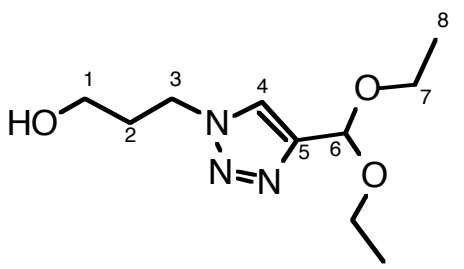

Prepared from $64 \mathrm{mg}(0.50 \mathrm{mmol})$ propiolaldehyde dimethyl acetal (4) and $56 \mathrm{mg}(0.55 \mathrm{mmol})$ propanol azide (7). The product 17 was obtained as a pale yellow oil (113 mg, $99 \%$ ). 
${ }^{1} \mathbf{H}$ NMR (CDCl $) \delta 1.22(\mathrm{t}, J=6.9 \mathrm{~Hz}, 6 \mathrm{H}, \mathrm{H}-8), 2.12(2 \mathrm{q}, J=6.0 \mathrm{~Hz}, 2 \mathrm{H}, \mathrm{H}-7), 2.74(\mathrm{~s}, 1 \mathrm{H}, \mathrm{OH}), 3.56-$ $3.70(\mathrm{~m}, 6 \mathrm{H}, \mathrm{H}-7), 4.51(\mathrm{t}, J=6.0 \mathrm{~Hz}, 2 \mathrm{H}, \mathrm{H}-3), 5.64$ (s, 1H, H-5), 7.64 (s, 1H, H-4)

${ }^{13} \mathbf{C ~ N M R}\left(\mathbf{C D C l}_{3}\right) \delta 15.1(\mathrm{C}-8), 32.6(\mathrm{C}-2), 47.1$ (C-3), 58.4 (C-1), $61.6(\mathrm{C}-7), 96.8$ (C-6), 122.3 (C-4), $147.0(\mathrm{C}-5)$

LC-MS : ELSD $95 \%, \mathrm{R}_{\mathrm{t}}=3.23 \mathrm{~min} ., \mathrm{m} / \mathrm{z} 252(\mathrm{M}+\mathrm{Na})^{+}, 481(2 \mathrm{M}+\mathrm{Na})^{+}$

\section{Methyl 1-(3-hydroxypropyl)triazole-4-carboxylate (18):}

$\mathrm{C}_{7} \mathrm{H}_{11} \mathrm{~N}_{3} \mathrm{O}_{3}, \mathrm{M}=185.18 \mathrm{~g} \cdot \mathrm{mol}^{-1}$

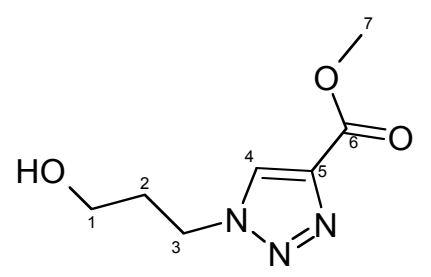

Prepared from $42 \mathrm{mg}(0.50 \mathrm{mmol})$ propiolic acid methyl ester (5) and $56 \mathrm{mg}(0.55 \mathrm{mmol})$ propanol azide (7). The product 18 was obtained as a beige solid (78 $\mathrm{mg}, 84 \%$ ).

$\mathrm{mp}=55-57^{\circ} \mathrm{C}$

${ }^{1} \mathbf{H}$ NMR $\left(\mathbf{C D C l}_{3}\right) \delta 2.18(\mathrm{q}, J=6.0 \mathrm{~Hz}, 2 \mathrm{H}, \mathrm{H}-2), 3.67(\mathrm{t}, J=6.0 \mathrm{~Hz}, 2 \mathrm{H}, \mathrm{H}-1), 3.93(\mathrm{~s}, 3 \mathrm{H}, \mathrm{H}-7), 4.62$ $(\mathrm{t}, J=6.0 \mathrm{~Hz}, 2 \mathrm{H}, \mathrm{H}-3), 8.22(\mathrm{~s}, 1 \mathrm{H}, \mathrm{H}-4)$

${ }^{13} \mathbf{C ~ N M R}\left(\mathbf{C D C l}_{3}\right) \delta 32.4(\mathrm{C}-2), 47.5(\mathrm{C}-3), 52.2(\mathrm{C}-7), 58.3(\mathrm{C}-1), 128.2(\mathrm{C}-5), 139.7(\mathrm{C}-4), 161.2(\mathrm{C}-$ 6)

LC-MS : ELSD 99\%, $\mathrm{R}_{\mathrm{t}}=2.98 \mathrm{~min} ., \mathrm{m} / \mathrm{z} 186(\mathrm{M}+\mathrm{H})^{+}, 208(\mathrm{M}+\mathrm{Na})^{+}, 393(2 \mathrm{M}+\mathrm{Na})^{+}$

Ethyl 2-[4-[3-(1,3-dioxoisoindolin-2-yl)propyl]triazol-1-yl]acetate (19):

$\mathrm{C}_{15} \mathrm{H}_{14} \mathrm{~N}_{4} \mathrm{O}_{4}, \mathrm{M}=314.30$ g.mol ${ }^{-1}$

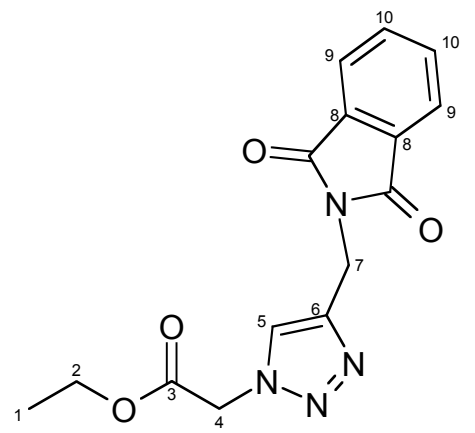

Prepared from $93 \mathrm{mg}(0.50 \mathrm{mmol})$ propargyl phthalimide (1) and $71 \mathrm{mg}(0.55 \mathrm{mmol})$ ethyl azidoacetate (8). The product 19 was obtained as a white solid (119 mg, $76 \%)$.

$\mathrm{mp}=55-57^{\circ} \mathrm{C}$

${ }^{1} \mathbf{H}$ NMR $\left(\mathbf{C D C l}_{3}\right) \delta 1.27(\mathrm{t}, J=7.2 \mathrm{~Hz}, 3 \mathrm{H}, \mathrm{H}-1), 4.23(\mathrm{q}, J=7.2 \mathrm{~Hz}, 2 \mathrm{H}, \mathrm{H}-2), 4.99$ (s, 2H, H-7), 5.11 (s, 2H, H-4), 7.70-7.82 (m, 5H, H-5, H-9, H-10)

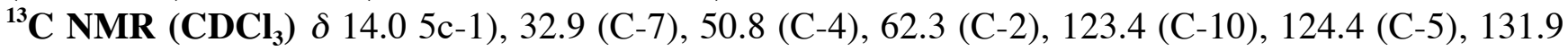
(11), 134.1 (C-9), 143.0 (C-6), 166.2 (C-3), 167.6 (C-8)

LC-MS : ELSD 91\%, $\mathrm{R}_{\mathrm{t}}=5.55 \mathrm{~min} ., \mathrm{m} / \mathrm{z} 315(\mathrm{M}+\mathrm{H})^{+}$ 
Ethyl 2-[4-(phenoxymethyl)triazol-1-yl]acetate (20):

$\mathrm{C}_{13} \mathrm{H}_{15} \mathrm{~N}_{3} \mathrm{O}_{3}, \mathrm{M}=261.28 \mathrm{~g} \cdot \mathrm{mol}^{-1}$<smiles>O=C(Cn1cc(COc2ccccc2)nn1)OCI</smiles>

Prepared from $66 \mathrm{mg}(0.50 \mathrm{mmol})$ propargyl phenyl ether (2) and $71 \mathrm{mg}(0.55 \mathrm{mmol})$ ethyl azidoacetate $(\mathbf{8})$. The product $\mathbf{2 0}$ was obtained as a pale yellow oil (129 $\mathrm{mg}, 99 \%)$.

${ }^{1} \mathbf{H}$ NMR $\left(\mathbf{C D C l}_{3}\right) \delta 1.25(\mathrm{t}, J=7.2 \mathrm{~Hz}, 3 \mathrm{H}, \mathrm{H}-1), 4.23$ (q, J=7.2 Hz, 2H, H-2), 5.11 (s, 2H, H-4), 5.18 (s, 2H, H-7), 6.92-7.29 (m, 5H, H-9,H-10,H-11), 7.73 (s, 1H, H-5)

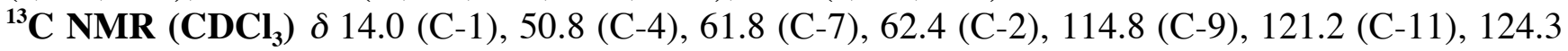
(C-5), 129.5 (C-10), 144.5 (C-6), 158.2 (C-8), 166.2 (C-3)

LC-MS : ELSD 97\%, $\mathrm{R}_{\mathrm{t}}=8.66 \mathrm{~min} ., \mathrm{m} / \mathrm{z} 262(\mathrm{M}+\mathrm{H})^{+}$

Ethyl 2-[4-(hydroxymethyl)triazol-1-yl]acetate (21):

$\mathrm{C}_{7} \mathrm{H}_{11} \mathrm{~N}_{3} \mathrm{O}_{3}, \mathrm{M}=185.18 \mathrm{~g} \cdot \mathrm{mol}^{-1}$

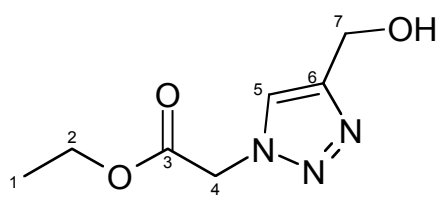

Prepared from $28 \mathrm{mg}(0.50 \mathrm{mmol})$ propargyl alcohol $(\mathbf{3})$ and $71 \mathrm{mg}(0.55 \mathrm{mmol})$ ethyl azidoacetate $(\mathbf{8})$. The product $\mathbf{2 1}$ was obtained as a pale yellow oil (92 $\mathrm{mg}, 99 \%$ ).

${ }^{1} \mathbf{H}$ NMR $\left(\mathbf{C D C l}_{3}\right) \delta 1.26(\mathrm{t}, J=7.2 \mathrm{~Hz}, 3 \mathrm{H}, \mathrm{H}-1), 4.21$ (q, J=7.2 Hz, 2H, H-2), 4.72 (s, 2H, H-7), 5.12 (s, 2H, H-4), 7.67 (s, 1H, H-5)

${ }^{13} \mathbf{C ~ N M R}\left(\mathbf{C D C l}_{3}\right) \delta 14.0(\mathrm{C}-1), 50.8(\mathrm{C}-4), 56.1$ (C-2), 62.4 (C-7), 123.8 (C-5), $148.3(\mathrm{C}-6), 166.5$ (C3)

LC-MS : ELSD 99\%, $\mathrm{R}_{\mathrm{t}}=3.02 \mathrm{~min} ., \mathrm{m} / \mathrm{z} 186(\mathrm{M}+\mathrm{H})^{+}, 208(\mathrm{M}+\mathrm{Na})^{+}$

Ethyl 2-[4-(diethoxymethyl)triazol-1-yl]acetate (22):

$\mathrm{C}_{11} \mathrm{H}_{19} \mathrm{~N}_{3} \mathrm{O}_{4}, \mathrm{M}=257.29 \mathrm{~g} \cdot \mathrm{mol}^{-1}$

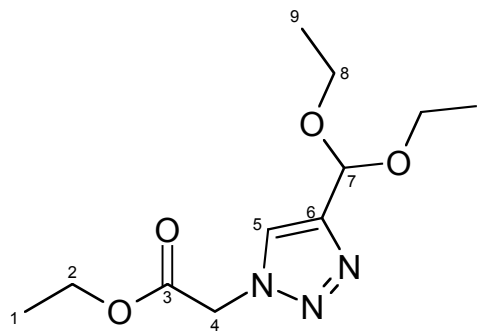

Prepared from $64 \mathrm{mg}(0.50 \mathrm{mmol})$ propiolaldehyde dimethyl acetal (4) and $71 \mathrm{mg}(0.55 \mathrm{mmol})$ ethyl azidoacetate (8). The product 22 was obtained as a pale yellow oil (127 $\mathrm{mg}, 99 \%)$. 
${ }^{1} \mathbf{H}$ NMR (CDCl $) \delta 1.12-1.22(\mathrm{~m}, 9 \mathrm{H}, \mathrm{H}-1, \mathrm{H}-9), 3.57(2 \mathrm{q}, J=6.9 \mathrm{~Hz}, 4 \mathrm{H}, \mathrm{H}-8), 4.18(\mathrm{q}, J=7.2 \mathrm{~Hz}, 2 \mathrm{H}$, H-2), 5.09 (s, 2H, H-4), 5.67 (s, 1H, H-7), 7.68 (s, 1H, H-5)

${ }^{13} \mathbf{C ~ N M R}\left(\mathbf{C D C l}_{3}\right) \delta 14.4(\mathrm{C}-1), 15.5(\mathrm{C}-9), 51.2(\mathrm{C}-4), 61.8$ (C-8), $62.7(\mathrm{C}-2), 97.0(\mathrm{C}-7), 124.0(\mathrm{C}-5)$, 148.1 (C-6), $166.2(\mathrm{C}-3)$

LC-MS : ELSD 99\%, $\mathrm{R}_{\mathrm{t}}=5.36 \mathrm{~min} ., \mathrm{m} / \mathrm{z} 212(\mathrm{M}-\mathrm{EtO}), 280(\mathrm{M}+\mathrm{Na})^{+}$

Methyl 1-(ethoxycarbonylmethyl)triazole-4-carboxylate (23):

$\mathrm{C}_{8} \mathrm{H}_{11} \mathrm{~N}_{3} \mathrm{O}_{4}, \mathrm{M}=213.19 \mathrm{~g} \cdot \mathrm{mol}^{-1}$

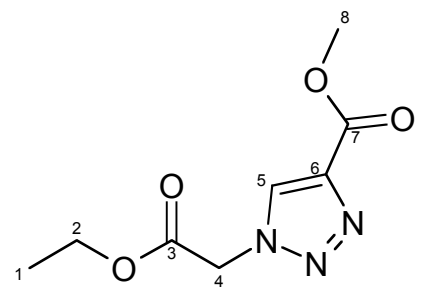

Prepared from $42 \mathrm{mg}(0.50 \mathrm{mmol})$ propiolic acid methyl ester (5) and $71 \mathrm{mg}(0.55 \mathrm{mmol})$ ethyl azidoacetate (8). The product $\mathbf{2 3}$ was obtained as a beige solid (105 $\mathrm{mg}, 99 \%)$.

$\mathrm{mp}=102-104{ }^{\circ} \mathrm{C}$

${ }^{1} \mathbf{H} \mathbf{N M R}\left(\mathbf{C D C l}_{3}\right) \delta 1.30(\mathrm{t}, J=7.2 \mathrm{~Hz}, 3 \mathrm{H}, \mathrm{H}-1), 3.95(\mathrm{~s}, 3 \mathrm{H}, \mathrm{H}-5), 4.28(\mathrm{q}, J=7.2 \mathrm{~Hz}, 2 \mathrm{H}, \mathrm{H}-2), 5.25$ (s, 2H, H-4), 8.30 (s, 1H, H-5)

${ }^{13} \mathbf{C ~ N M R}\left(\mathbf{C D C l}_{3}\right) \delta 14.4(\mathrm{C}-1), 51.4(\mathrm{C}-4), 52.7$ (C-8), $63.2(\mathrm{C}-2), 129.4(\mathrm{C}-5), 161.4(\mathrm{C}-7), 166.0(\mathrm{C}-$ $3)$.

LC-MS : ELSD 91\%, $\mathrm{R}_{\mathrm{t}}=3.68 \mathrm{~min} ., \mathrm{m} / \mathrm{z} 214(\mathrm{M}+\mathrm{H})^{+}$ 
2-(1-Benzyl-1H-[1,2,3]triazol-4-ylmethyl)-isoindole-1,3-dione (9) :
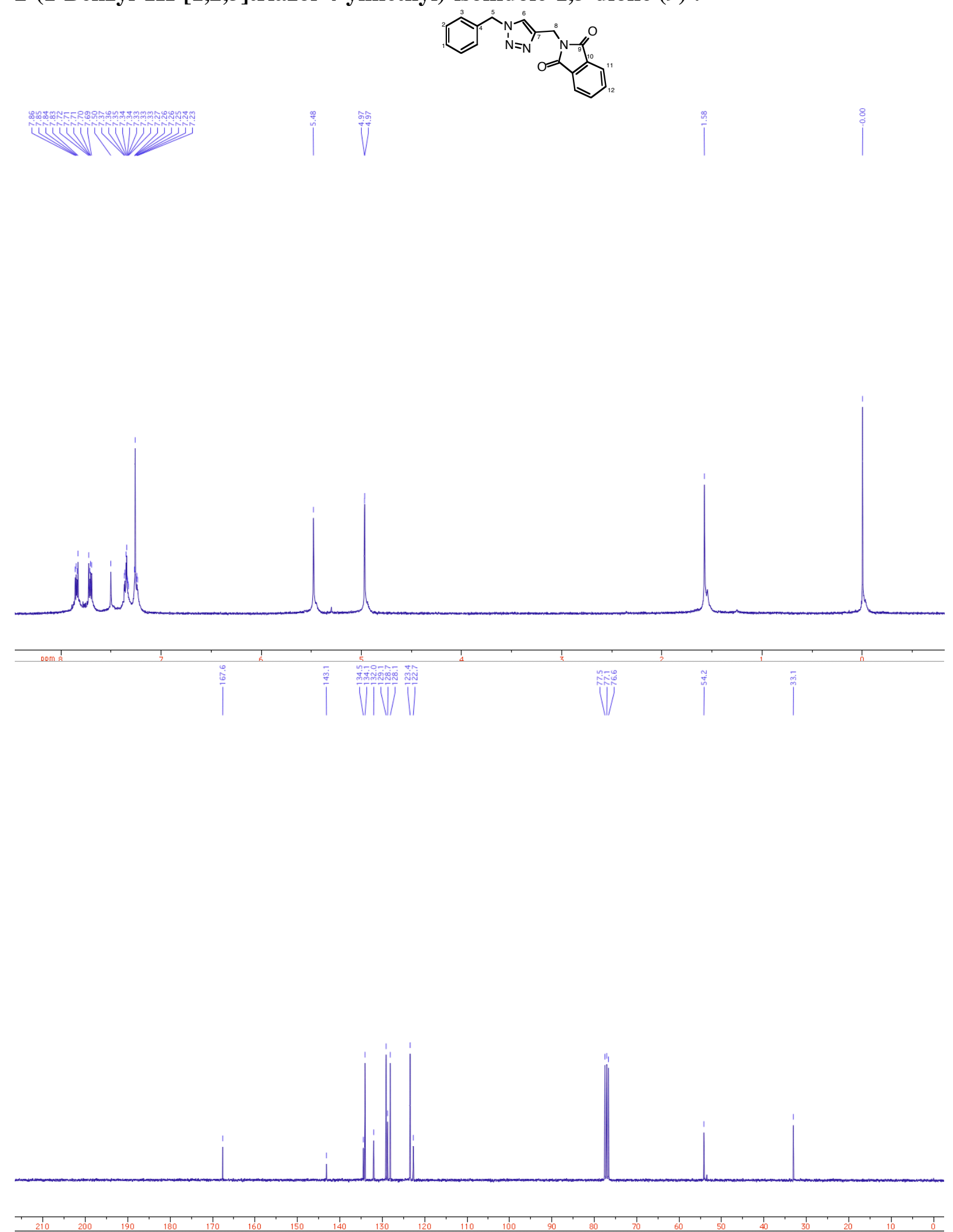
1-benzyl-4-(phenoxymethyl)triazole (10):

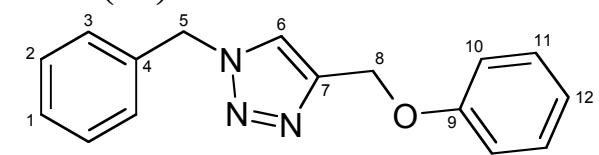

s:
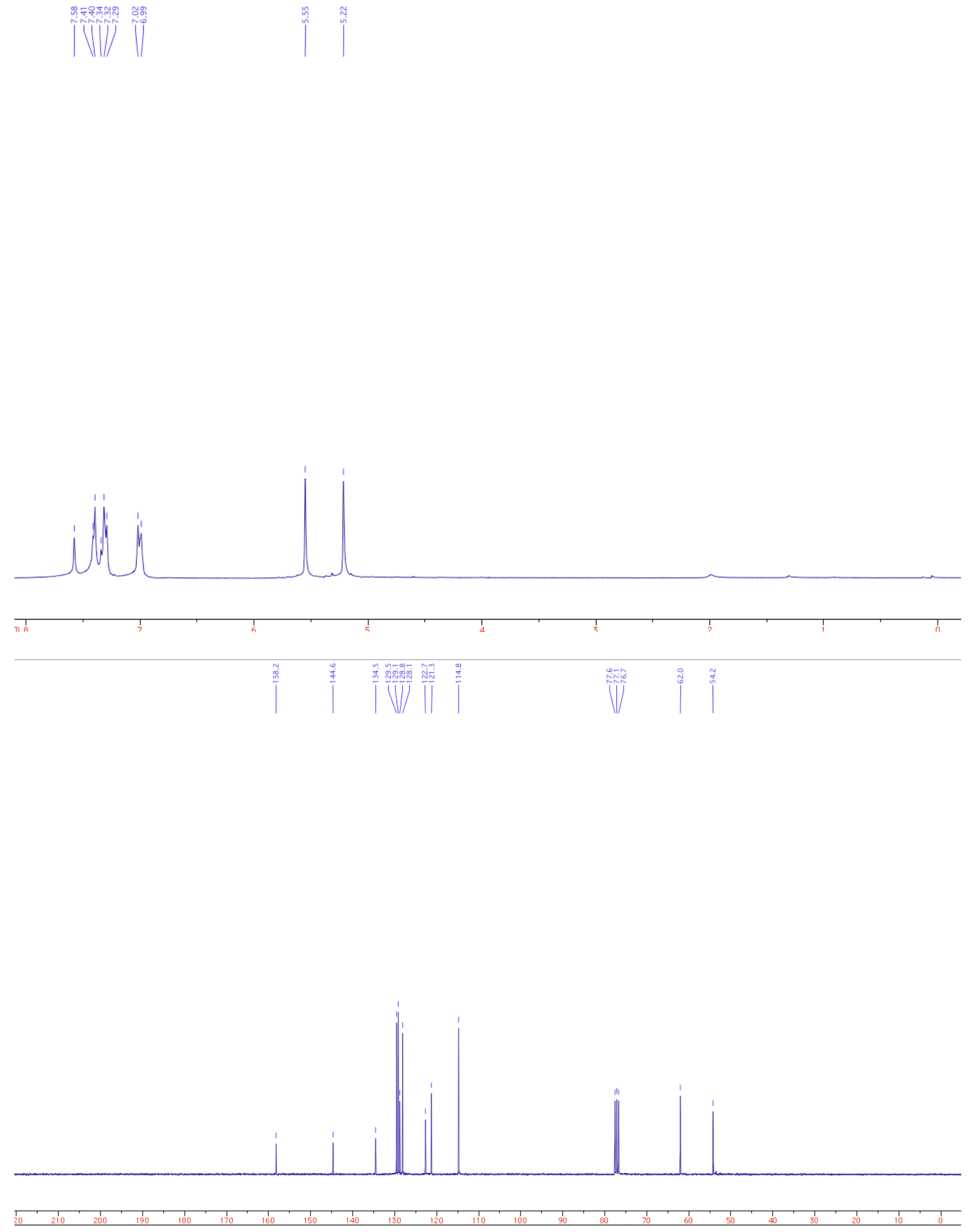

S-10 
(1-benzyltriazol-4-yl)methanol (11) :<smiles>OCc1cn(Cc2ccccc2)nn1</smiles>

IVII
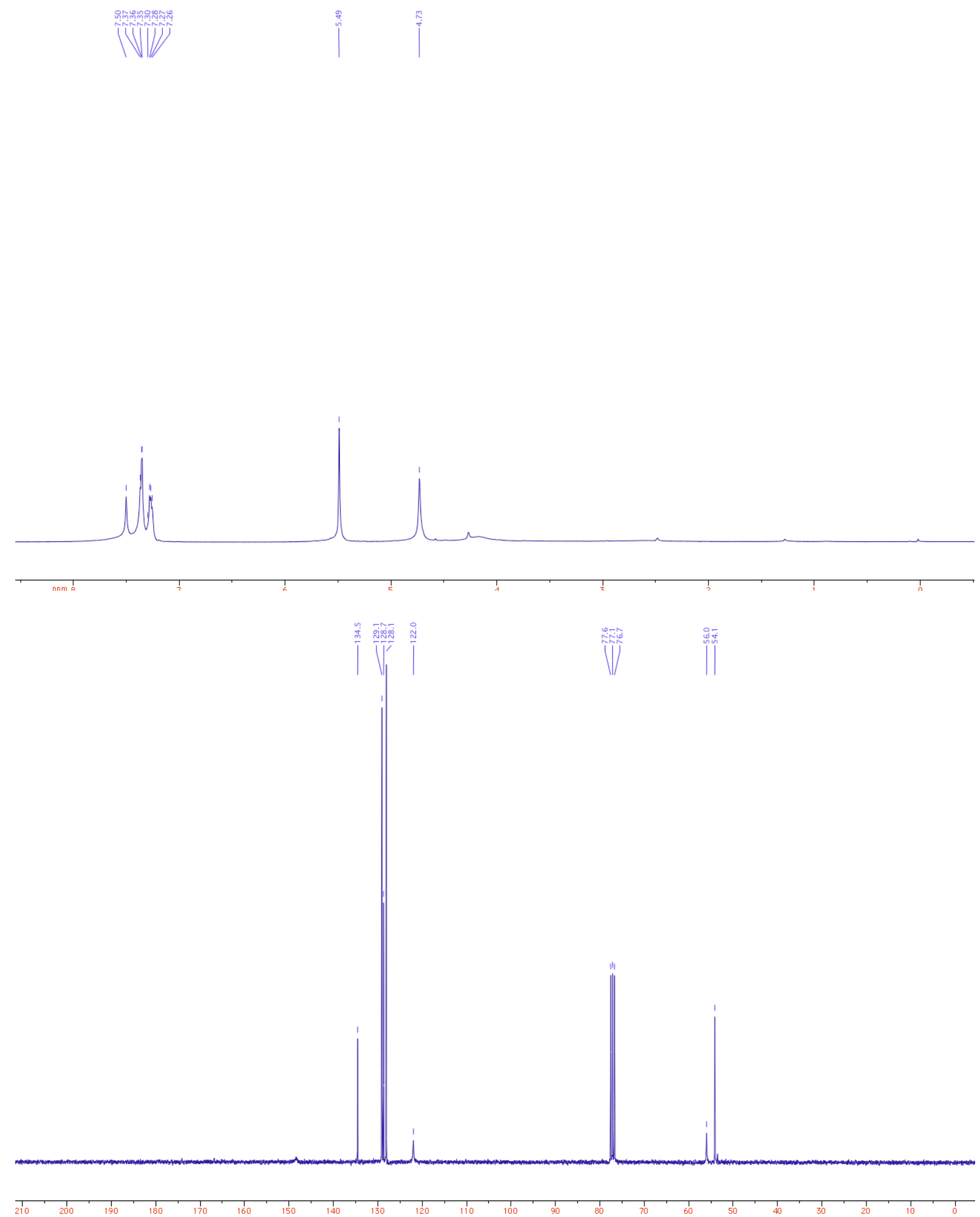
1-benzyl-4-(diethoxymethyl)triazole (12) :
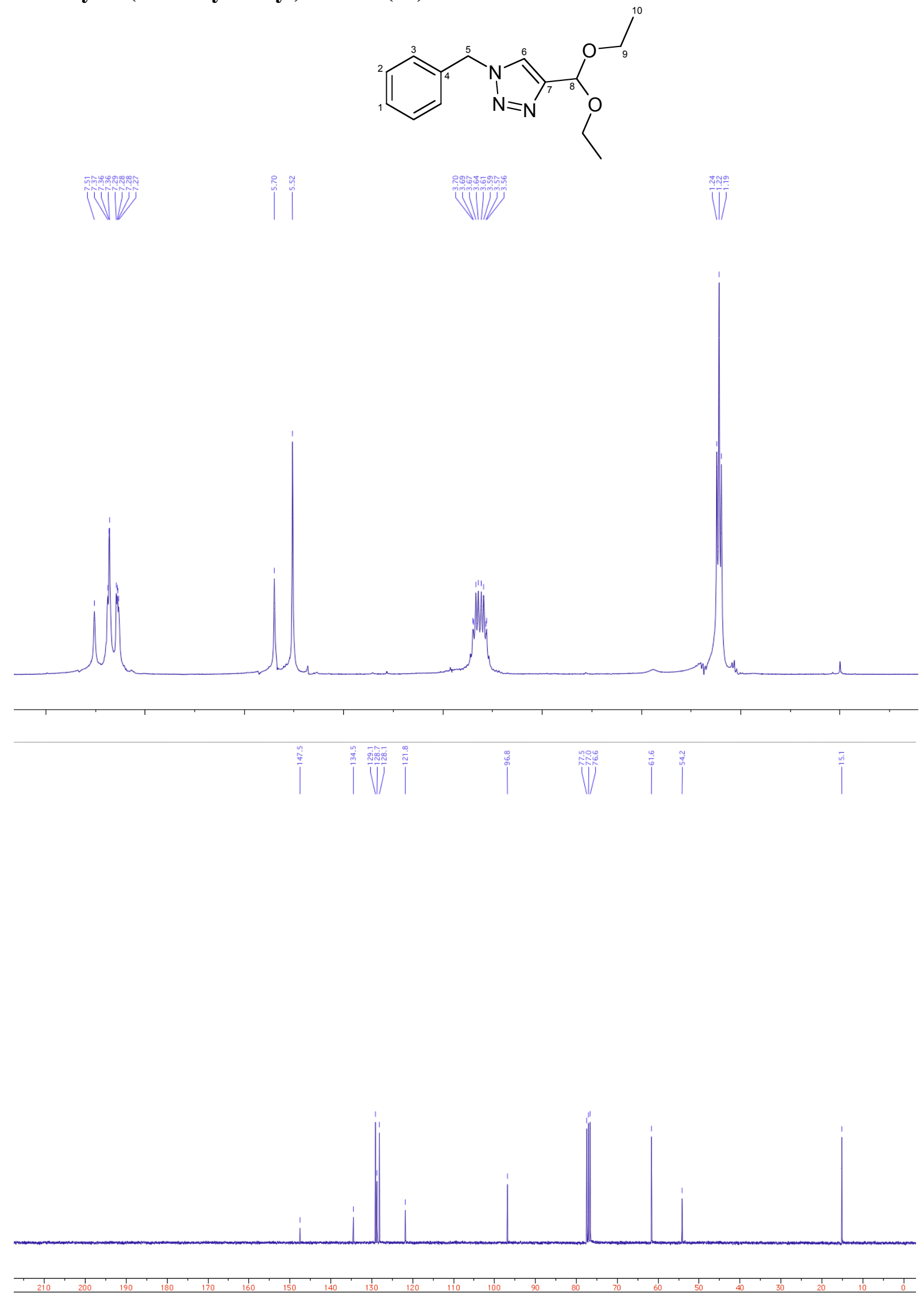

S-12 
Methyl 1-benzyltriazole-4-carboxylate (13) :<smiles>COC(=O)c1cn(Cc2ccccc2)nn1</smiles>
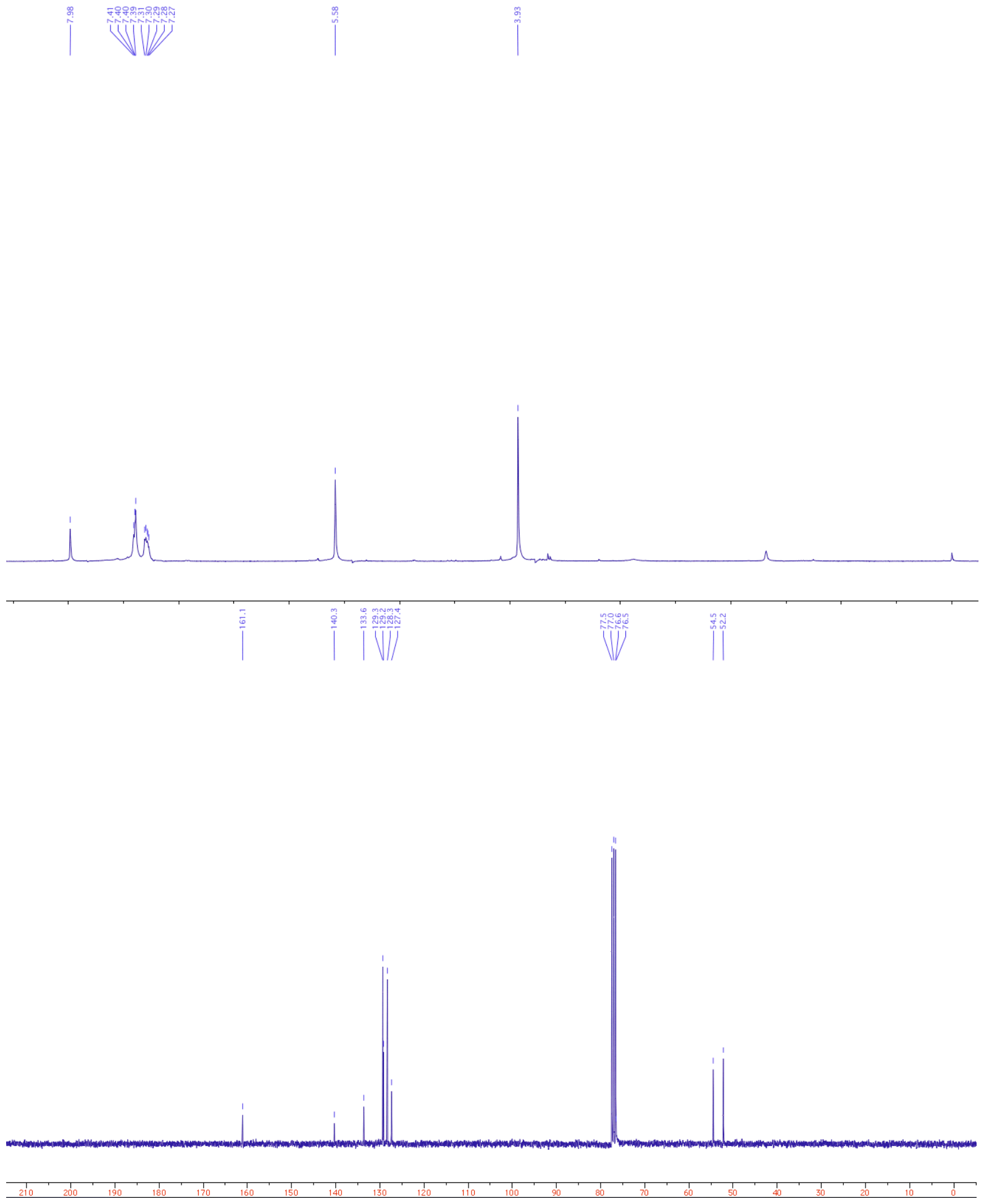

S-13 
3-(4-phenoxytriazol-1-yl)propan-1-ol (14) :

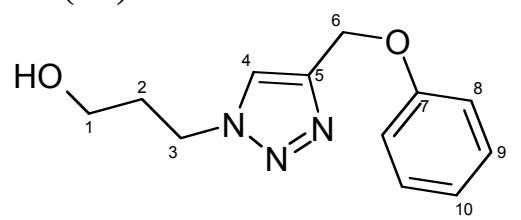

W

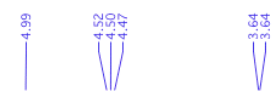

Viv

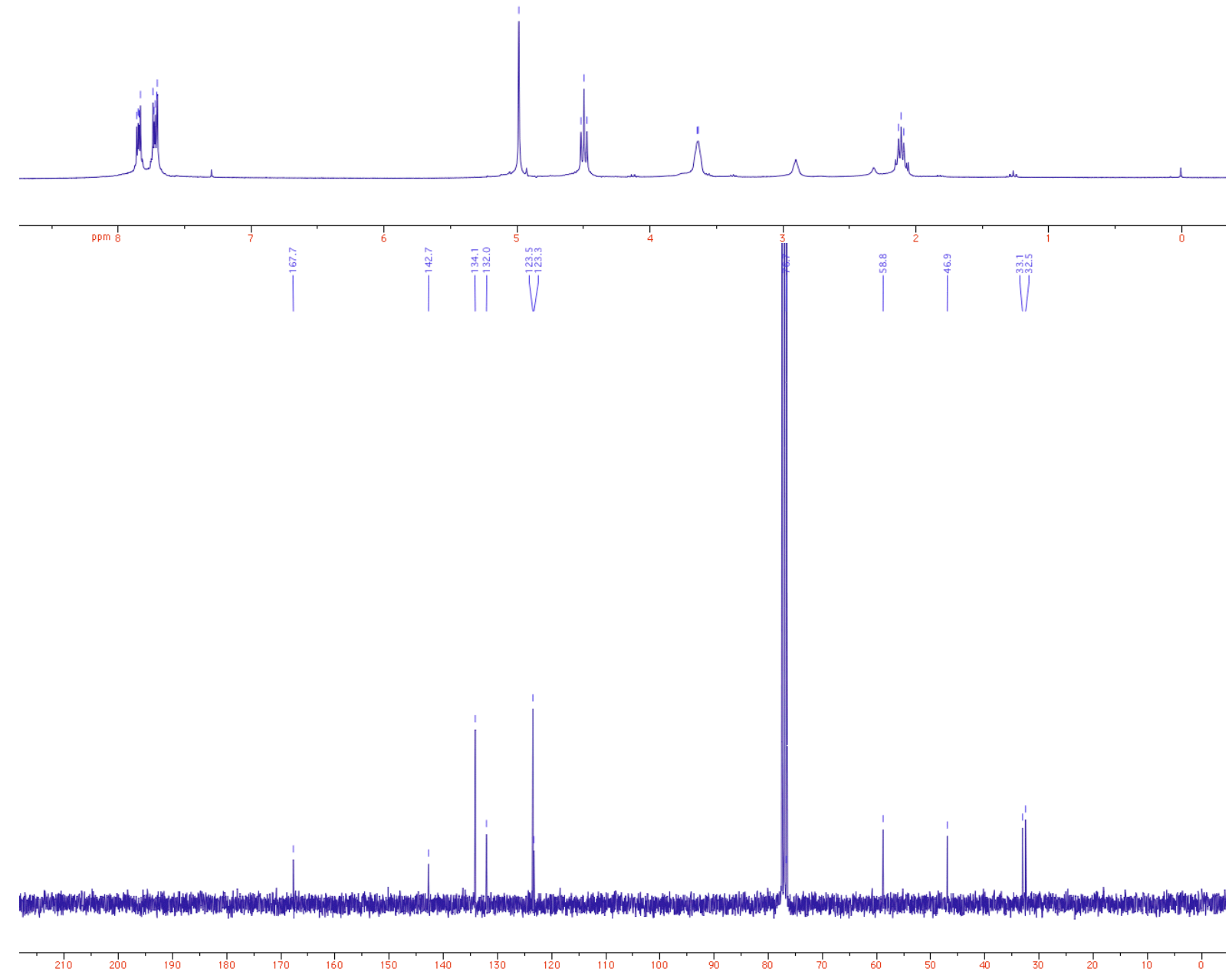

S-14 
3-(4-phenoxytriazol-1-yl)propan-1-ol (15) :
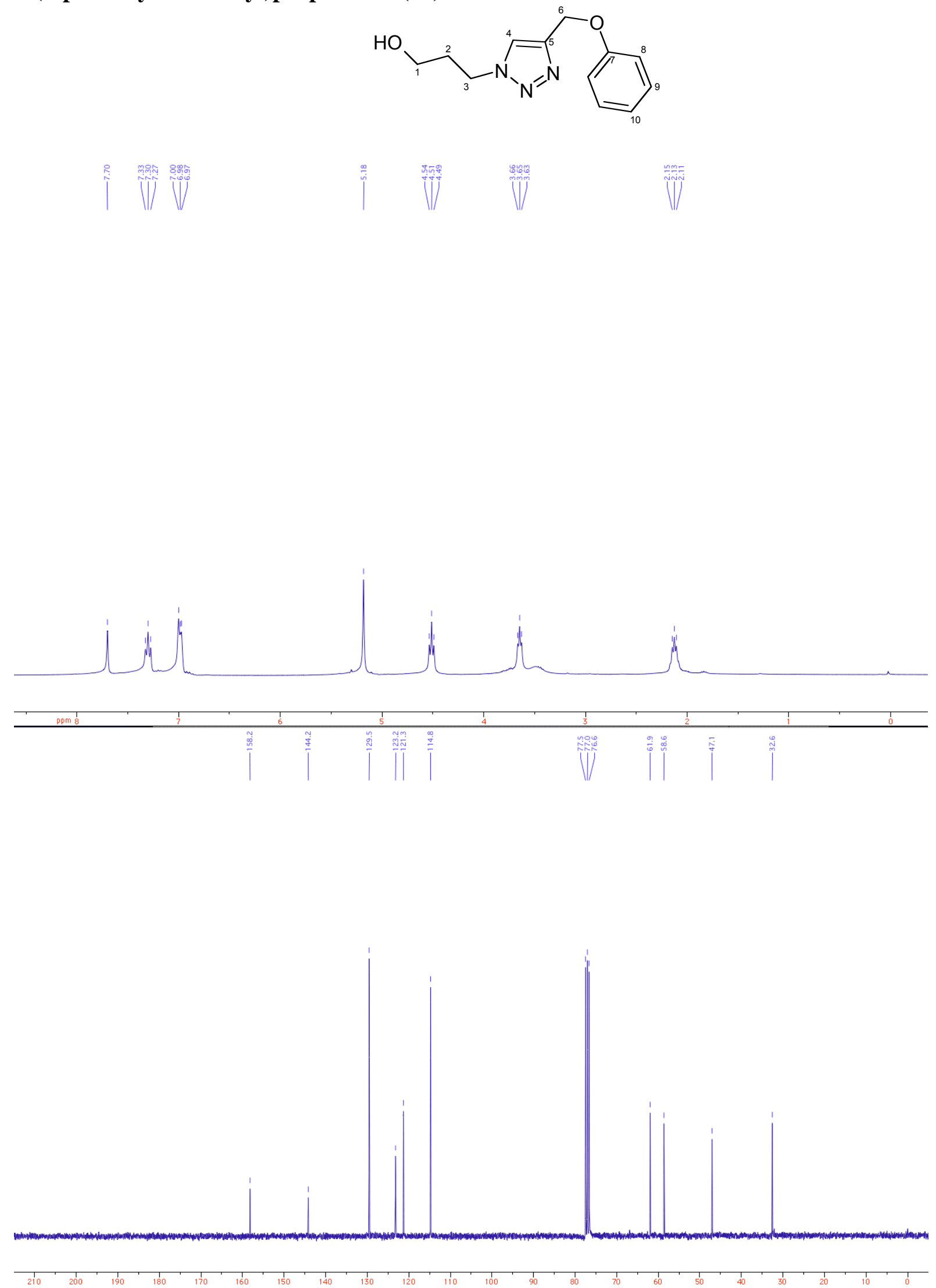
3-[4-(hydroxymethyl)triazol-1-yl]propan-1-ol (16) :
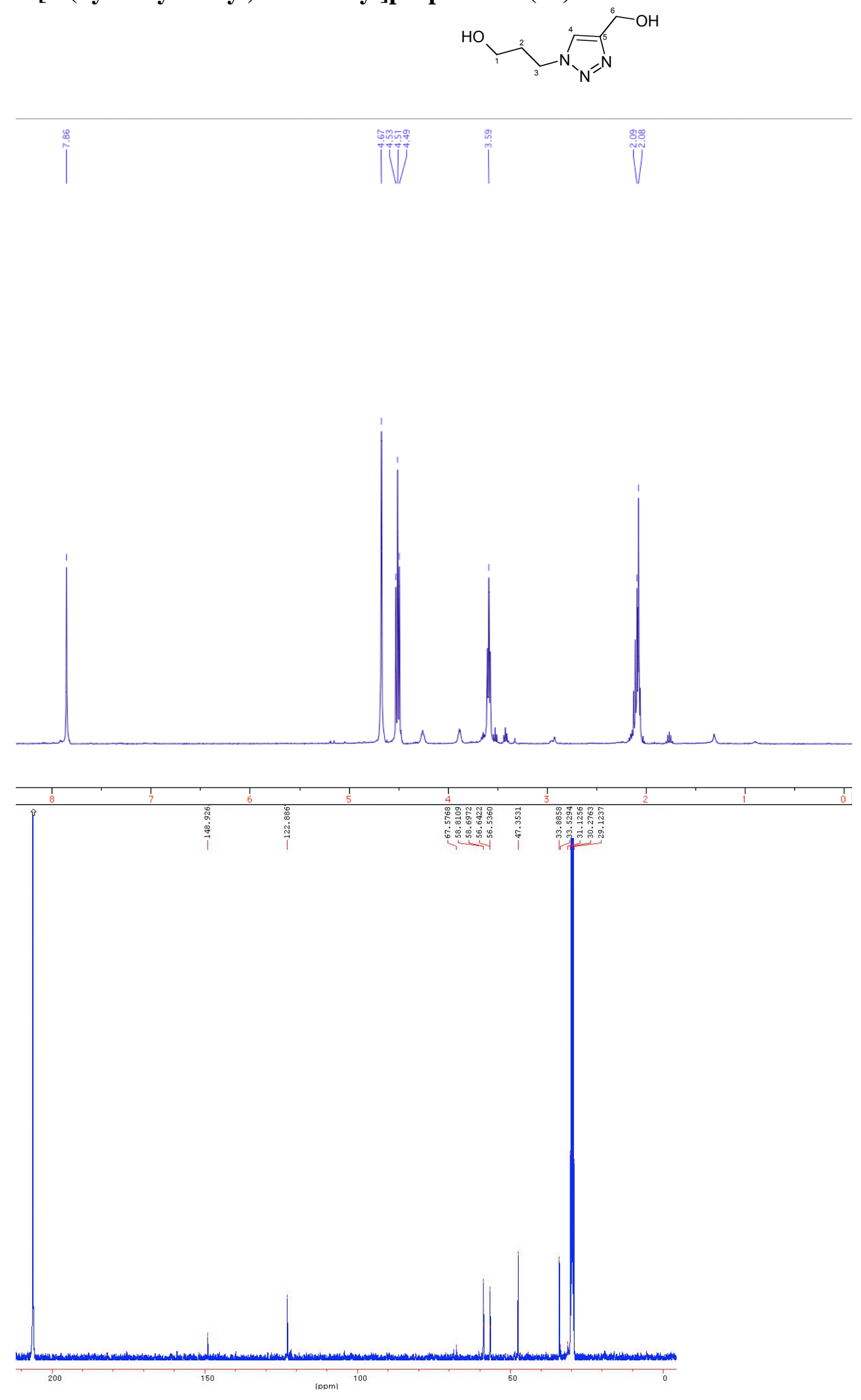

S-16 
3-[4-(diethoxymethyl)triazol-1-yl]propan-1-ol (17) :
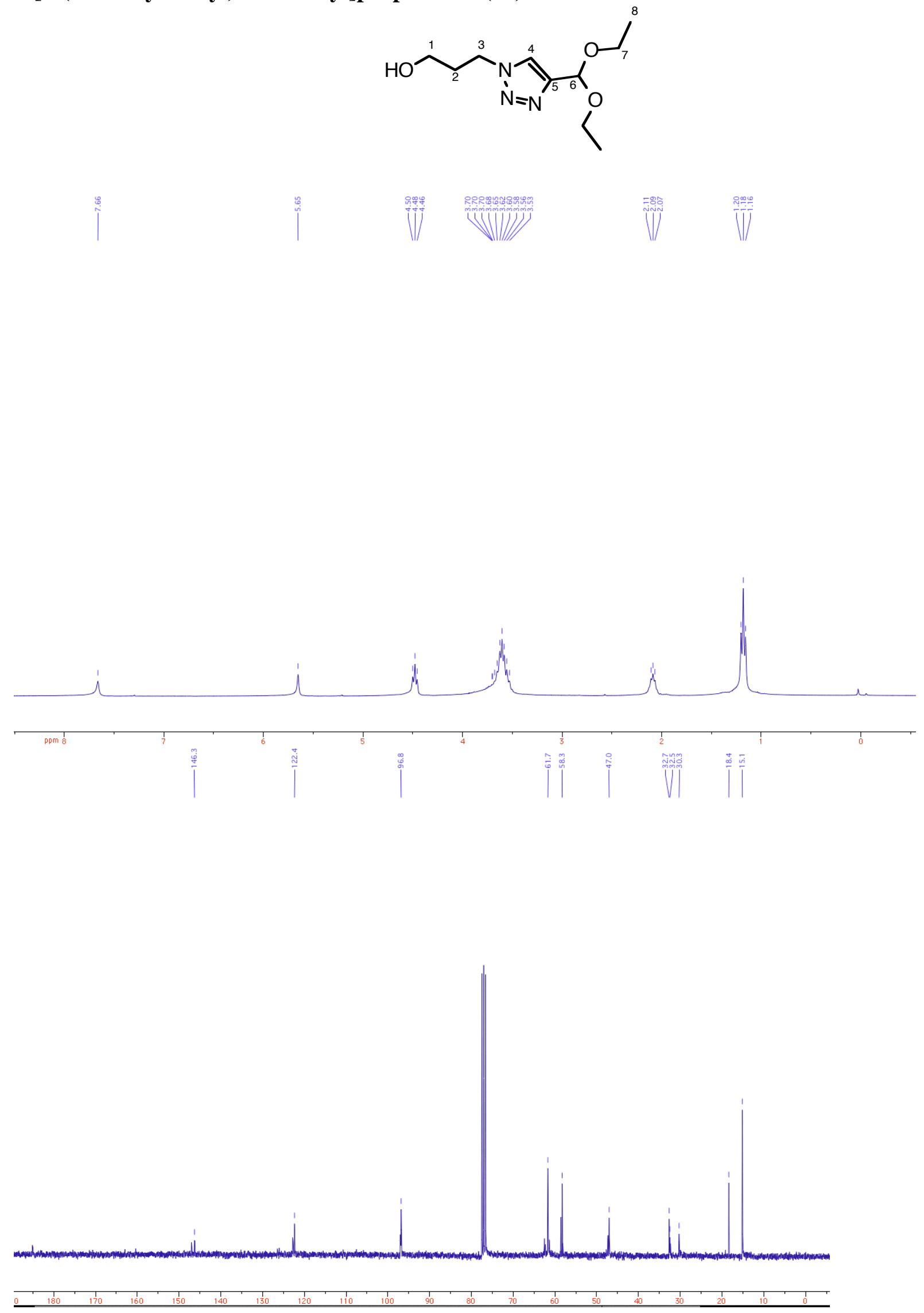

S-17 
Methyl 1-(3-hydroxypropyl)triazole-4-carboxylate (18) :
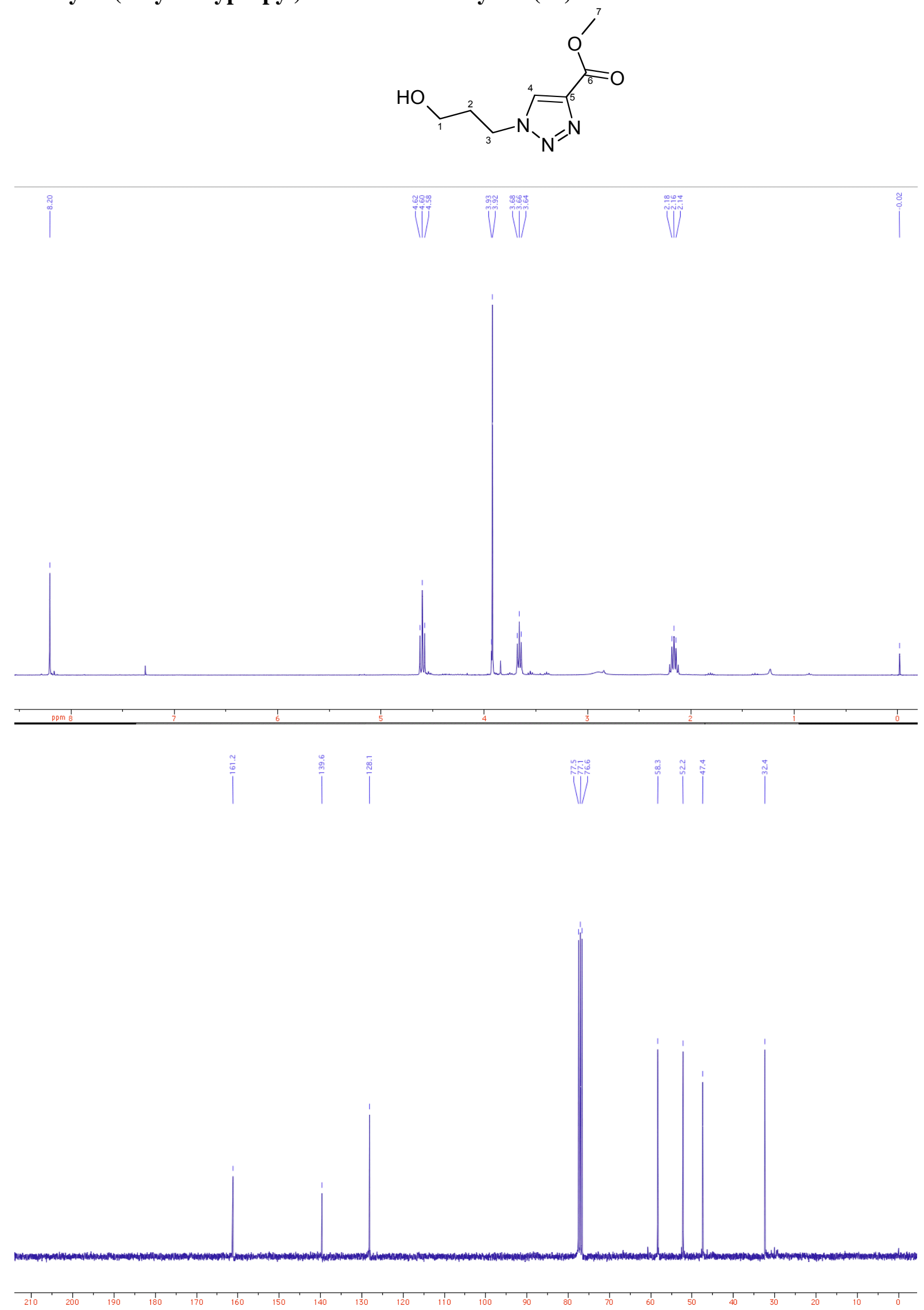

S-18 
Ethyl 2-[4-[3-(1,3-dioxoisoindolin-2-yl)propyl]triazol-1-yl]acetate (19) :
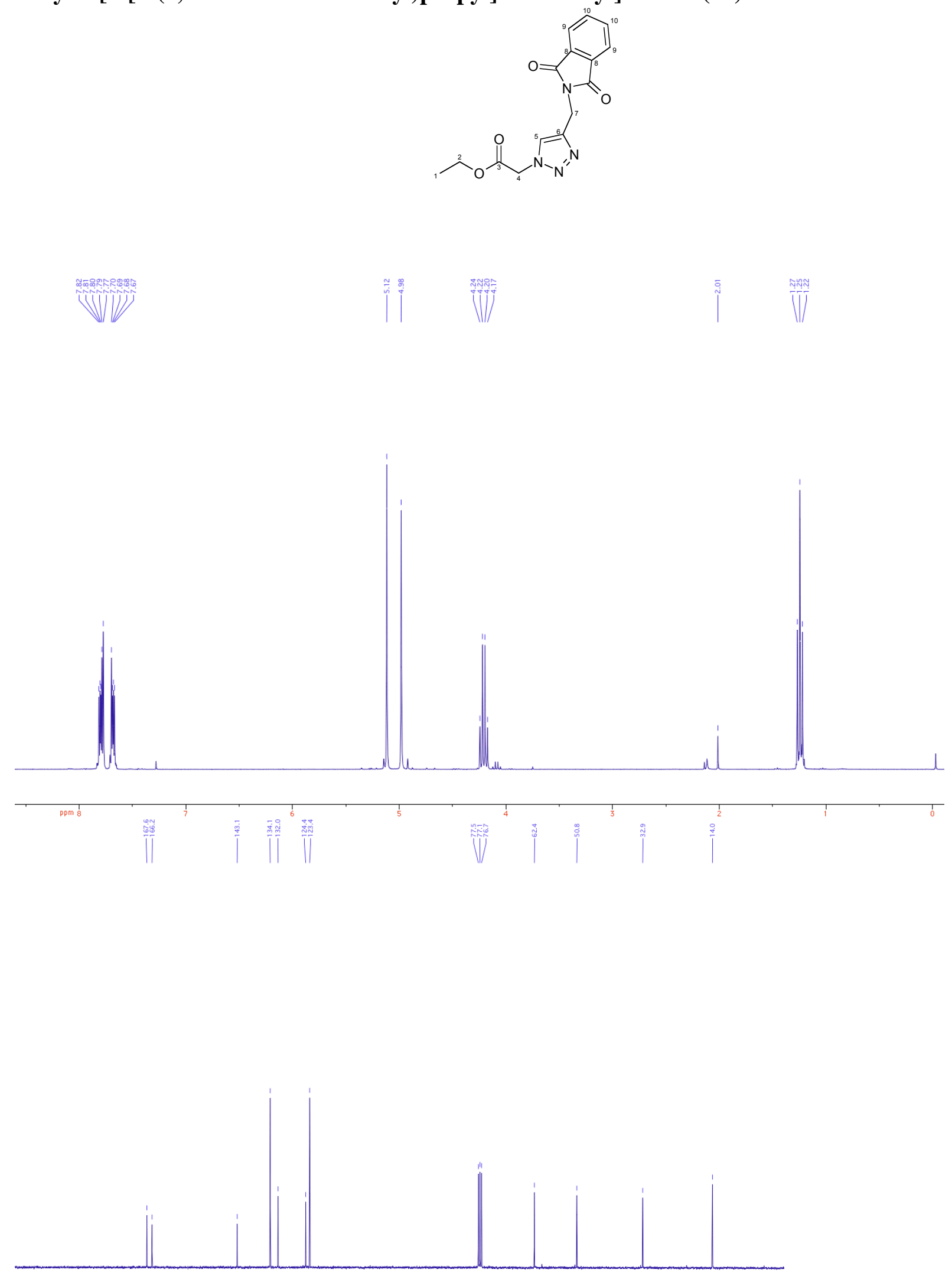

S-19 
Ethyl 2-[4-(phenoxymethyl)triazol-1-yl]acetate (20) :
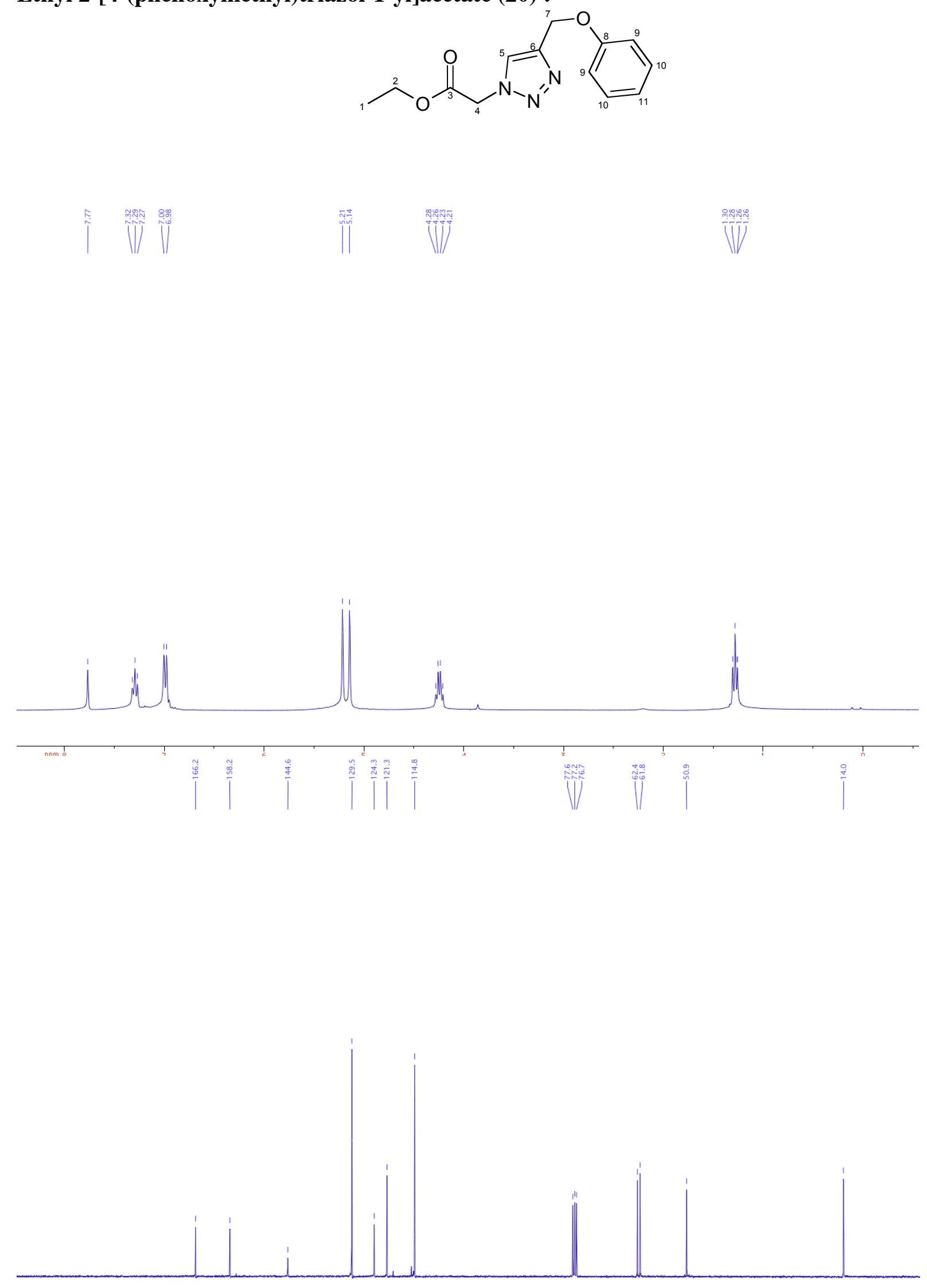
Ethyl 2-[4-(hydroxymethyl)triazol-1-yl]acetate (21) :<smiles>CCOC(=O)Cn1cc(CO)nn1</smiles>
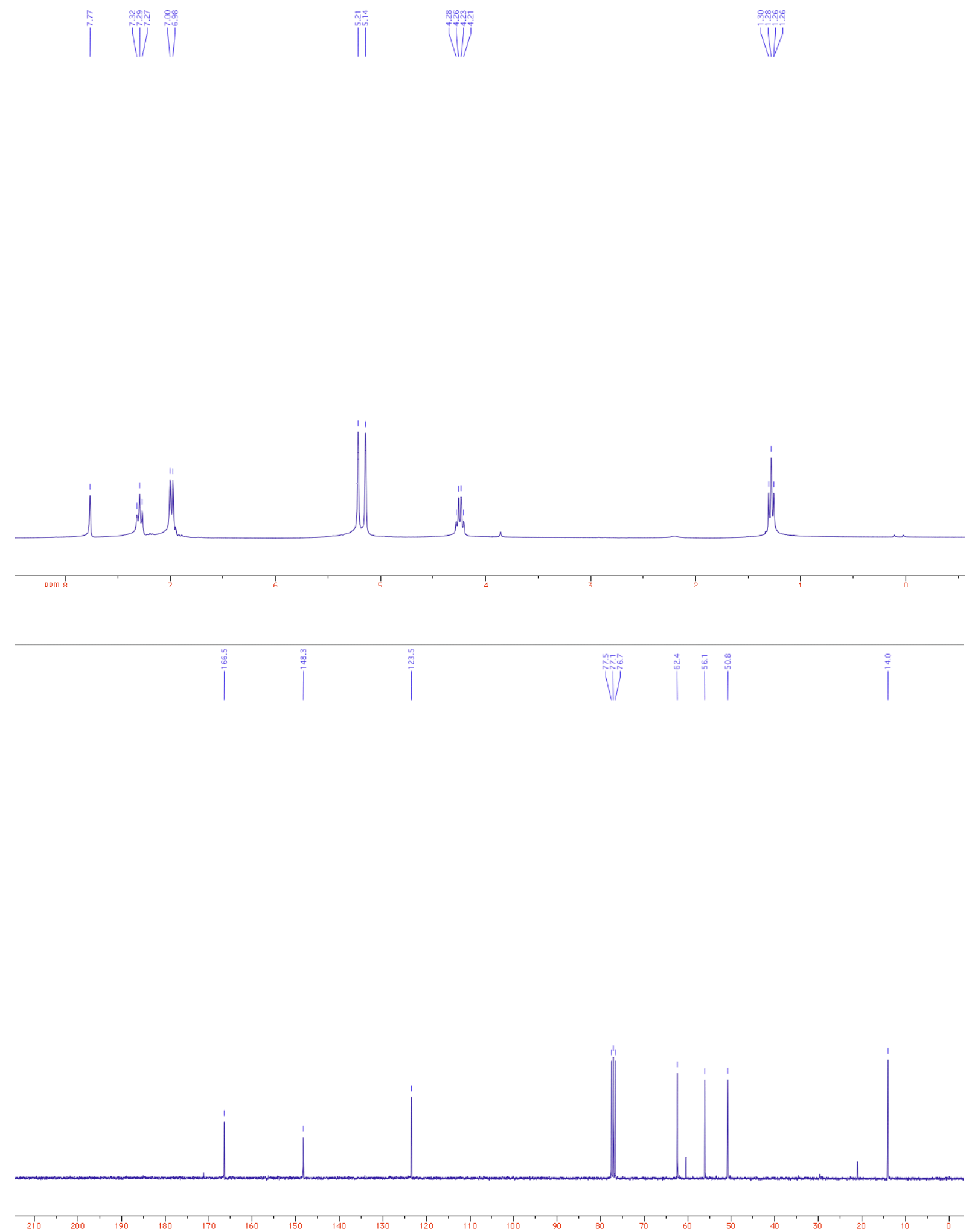

S-21 
Ethyl 2-[4-(diethoxymethyl)triazol-1-yl]acetate (22) :
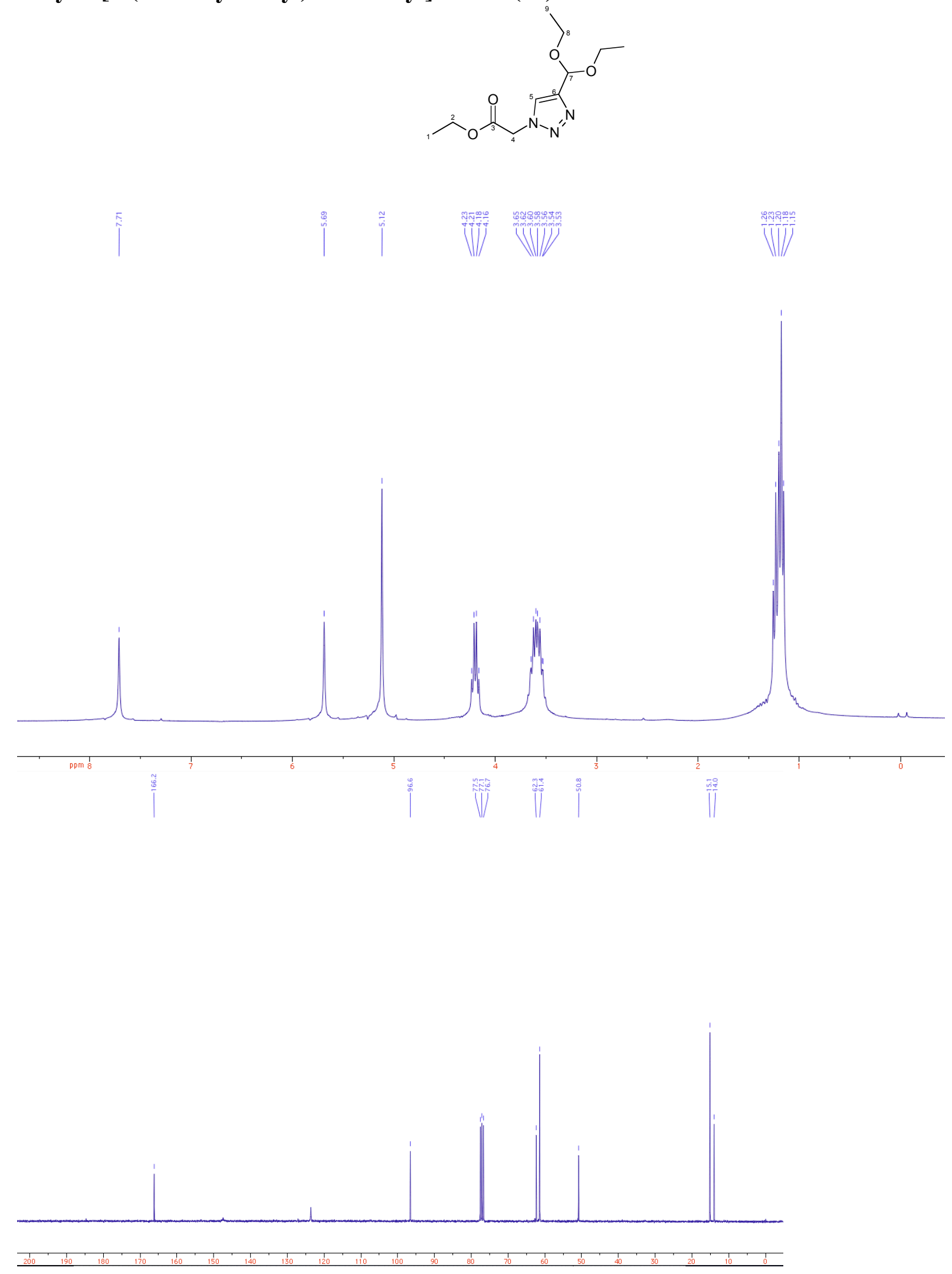
Methyl 1-(ethoxycarbonylmethyl)triazole-4-carboxylate (23) :
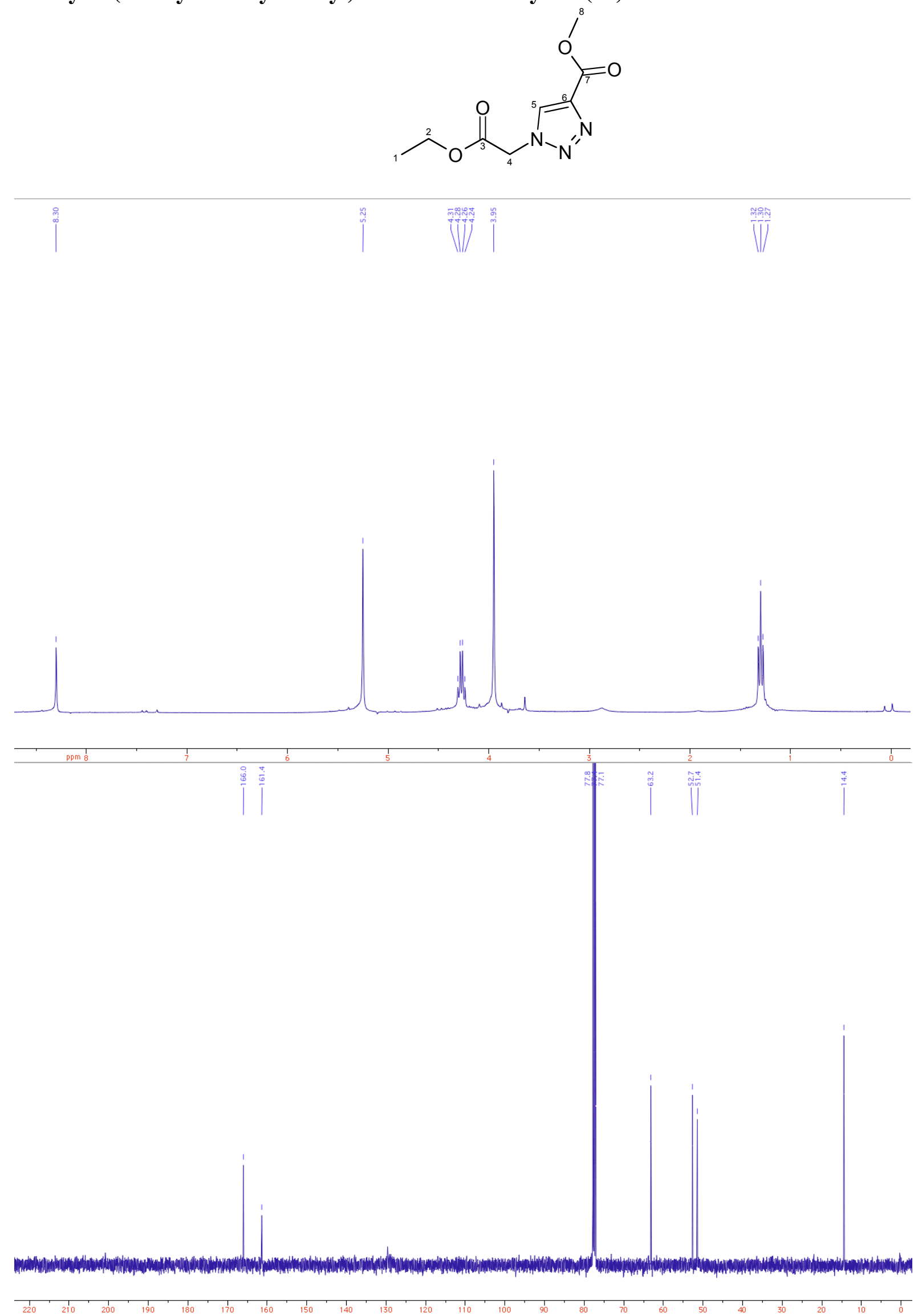

S-23 